\title{
Local knowledge about fodder plants in the semi-arid region of Northeastern Brazil
}

\author{
Alissandra Trajano Nunes ${ }^{1}$, Reinaldo Farias Paiva de Lucena ${ }^{2}$, Mércia Virgínia Ferreira dos Santos ${ }^{3}$ \\ and Ulysses Paulino Albuquerque ${ }^{1 *}$
}

\begin{abstract}
Background: This study evaluated local knowledge of the fodder plants of the Caatinga in northeast Brazil (seasonal dry forest). Specifically, the goal was to catalog local knowledge regarding the use of native and exotic forage plants in two rural communities located in the state of Paraíba (northeast Brazil), to provide information for nutritional investigations and to verify how the knowledge of these resources is distributed.

Methods: The communities were followed for three consecutive years, and interviews were conducted with 44 families (20 men and 24 women). Nine of these individuals were determined by the snowball technique to be key informants who held more specific knowledge about the topic. The data were structured into a database and statistically analyzed.

Results: Overall, 136 plants from 37 families and 113 genera were cited, and the knowledge of men was at a higher level than that of women $(p<0.05)$. Participants demonstrated a sophisticated knowledge of nutritional characteristics such as nutritional value, palatability, availability and productivity. Native plants were highlighted over the exotic, especially for species of the families Cactaceae, Bromeliaceae and Fabaceae.

Conclusions: The great diversity of plants cited by the informants demonstrates the potential of local vegetation and the importance of traditional knowledge in the research process and in the characterization of forage resources. This diversity also favors the selection of promising species for future biotechnological investigations.
\end{abstract}

Keywords: Ethnobotany, Ethnobiology, Animal feeding plants, Subsistence livestock

\section{Introduction}

Animal breeding is an ancient practice that represents an important source of subsistence for low-income households worldwide [1]. In Brazil, this activity plays an important role in the local economy, especially in the region of northeastern Brazil, where it accounts for more than $90 \%$ of the national flock of small ruminants, such as goats and sheep [2]. In this region, more than $50 \%$ of the territory is occupied by typical Caatinga vegetation (seasonal dry forest), which is the largest source of food for these animals [3].

Although it has been recently devastated, the Caatinga offers a good diversity of potential plants for the diet of ruminants $[4,5]$. However, the region, which is characterized

\footnotetext{
* Correspondence: upa@db.ufrpe.br

'Laboratório de Etnobiologia Aplicada e Teórica (LEA), Departamento de Biologia da Universidade Federal Rural de Pernambuco, Recife, Pernambuco CEP: 52171-900, Brazil

Full list of author information is available at the end of the article
}

by a semi-arid climate, has suffered environmental pressures and is affected by a strong influence of climatic seasonality that limits the productive and nutritional potential of the vegetation. The result is that it is difficult to maintain flocks, especially during drought periods $[4,6]$.

The situation in northeast Brazil mainly affects small farmers and is common in other countries with similar climatic conditions, such as Nigeria and Pakistan [7-9]. Given the growing need to produce human food and to secure subsistence food sources for flocks, there is a growing interest in expanding the knowledge about the fodder plants in these areas [1,7-11]. The investigation has advanced in the Caatinga $[4,5]$ to characterize the local natural resources and determine alternatives that may ease the difficulties of people who depend on subsistence farming to survive $[12,13]$.

In the investigation of food sources in different regions of the world, it is essential to document local knowledge 
as a valuable resource in the characterization of fodder plants $[11,14,15]$. This information can be captured in local history as well as by the indications of fodder uses in the lists of plants that were generated by ethnobotanical research conducted in the Northeastern countryside [16-18].

With respect to ethnobotanical approaches in northeastern Brazil, there is a gap related to the forage resource evidence in relation to reports published by researchers from other countries $[7,14,19,20]$. Consideration should be given to prioritizing access to local knowledge to combine scientific knowledge and optimize the process of characterizing the diversity of plants useful for this purpose.

In the last few decades, the union of scientific knowledge with traditional knowledge has been increased through several approaches. This union has revealed the importance of local wisdom in conservation as well as in providing basic information for bioprospecting [21-23].

Ethnobotanical investigations of fodder plants have been developed in African countries such as Ethiopia, Nigeria and Uganda, and elsewhere in Asia, India, and Mexico. These works are guided by approaches that reinforce the importance of local knowledge associated with the use, classification and management of useful plants in animal feed, and in seeking alternatives to reconcile sustainability and conservation of the local biodiversity see $[7,14,19]$.

This research aims to analyze the traditional knowledge about the plants used in animal feeding in two rural communities in northeastern Brazil. The work is based on the premise that rural communities in semiarid environments have an expansive knowledge of the diversity and nutritional potential of plants used as ruminant feed. Our main goals were to identify the species used in animal diets mentioned in both communities, check for differences in the biogeographical origins of the plants and identify the influence of seasonality in the diets of the animals.

Because animal husbandry is an activity performed by men, we evaluated whether there was a difference in knowledge between men and women. Therefore, we evaluated the responses by gender and correlated variables such as age, income and education with the use of fodder plants.

\section{Materials and methods Area of study}

The present study has been developed in the semi-arid region of Northeastern Brazil. The region is home to the Caatinga biome, which is considered to be the only Brazilian biome that is exclusive to Brazil. The biome shows distinct edaphoclimatic conditions that create units of differentiated landscape. This situation gives rise to diverse vegetation that is represented by a considerable richness of endemic species [3].

In terms of occupation, the region covers approximately $10 \%$ of the national territory and more than $80 \%$ of the Northeastern territory, with approximately $970,000 \mathrm{Km}^{2}$ of territorial extension inhabited by more than 20 million people [24].

A considerable part of the population lives in rural areas and historically is linked to agriculture, and particularly to livestock, which are better adapted than crops to climatic adversities [3]; this activity has a strong influence on the local and regional economy [2].

\section{Study area}

The study was carried out in the communities of Barrocas and Cachoeira, which are located in the rural zone around Soledade city $\left(7^{\circ} 03^{\prime} 26^{\prime \prime}\right.$ south latitude and $36^{\circ} 21^{\prime} 46^{\prime \prime}$ west longitude), in the state of Paraíba, in Northeastern Brazil. The region has a hot, semi-arid climate (BShs according to Köppen), with an average annual rainfall of $300 \mathrm{~mm}^{3}$ [25].

The Cachoeira and Barrocas communities are 14 and $18 \mathrm{~km}$ from the center of Soledade, respectively, and approximately $4.5 \mathrm{~km}$ from each other $[22,26]$. These communities have been selected based on prior ethnobotanical studies that have shown the population using vegetation resources for several purposes, including animal feeding $[22,26]$.

In Barrocas, 12 farms are registered and are located apart from each other with mostly rural properties. The properties range from 70 to 450 ha in area and are primarily used for animal breeding and the cultivation of forage cactus (Opuntia ficus-indica (L.) Mill.), corn (Zea mays L.) and beans (Phaseolus vulgaris L.). In Cachoeira, a total of 22 residences are registered close to each other, thereby forming a small village. Most of the residences have a backyard with space for animal breeding. The agricultural activities are shared in a land space of ordinary use, with approximately 70 ha devoted to subsistence agriculture and livestock $[22,26]$.

\section{Ethnobotanical survey}

The ethnobotanical survey was authorized by the Ethics Committee for Research Involving Human Beings of the Federal University of Pernambuco (register SISNEP FR 260099 and CEP/CCS/UFPE $\mathrm{N}^{\circ}$ 176/09) and was conducted between 2009 and 2011. All informants were guided to sign the free and clear consent form, which was a document that expressly showed that the signer was participating voluntarily.

Data collection used the technique of the free list, i.e., the free citation of species by the informant [23]. In this case, the following question was used: "Do you know plants for animal feeding (cattle, goats and sheep)?" 
From this list, the family heads responded to more detailed questions relating to knowledge of forage plants, such as the consumption, collection, quantity, nutrition, etc. This step was performed through interviews using semi-structured questionnaires to seek as much information as possible related to each species mentioned, as well as socioeconomic data such as education level, age, profession, monthly income, family composition, residence time and marital status. Finally, a screening was performed among respondents for the selection of key informants, i.e., those who have more knowledge on the subject. A third step of data collection was performed with these informants, in which all questions about each species were repeated to list species by the ordering technique [23]. In addition, questions were asked about plant parts, phenology, time of collection, amount collected, animal preference and nutritional attributes. To ensure the reliability of the information, interviews were conducted in two periods, in the early dry season (September-December 2009) and in the early rainy season (May-June 2010). At that time, participant observation was started, in which practices [23] during the collection activities of plants for animals were recorded, looking at the management of the animal in the pasture as well as diet and plants consumed in loco. These observations were captured in field diaries [23].

\section{Collection of plant material}

The collection and local identification of plants was performed with the help of local informers giving guided tours, in which more than one informant was invited to walk through the pastures and forests [23]. The species identification of the material was performed by specialists from the herbarium at the Agronomy Institute of Pernambuco (IPA).

\section{Data analysis}

The data achieved in the interviews were stored in a database in Microsoft Excel (Office 2007), and statistical analyses were performed with the BioEstat software (5.0) [27]. Each identified species was verified with regard to biogeographical origin, with species being considered either as native to South America or as exotic. The nomenclature and authorship of the species were updated in the database from the Missouri Botanical Garden (www.tropics.org) and the biogeographical origin through the consultation of the catalogue of plants and fungi, volumes I and II, of the Botanical Garden of Rio de Janeiro [28].

To evaluate the differences between the number of plants used and the number of plants known by the informants (those that effectively have indication of use, but in practice are not used every day by the informant), the Wilcoxon-Mann-Whitney test $(\mathrm{P}<0.05)$ was adopted.
The chi-square test (alpha $<0.05)$ was used to evaluate the differences between the richness of native and exotic plants in the studied communities.

The difference in richness of useful species during the drought and rain seasons was found through the chisquare test. The same test was adopted to verify if there were differences among the species cited in the communities.

The Jaccard coefficient (CJ) was used to verify the resemblance of species mentioned among the communities, adopting the formula $\mathrm{CJ}=\mathrm{a} / \mathrm{a}+\mathrm{b}+\mathrm{c}$, where $\mathbf{a}$ is the number of plants common to both communities; $\mathbf{b}$ is the total of species unique in the community a and $\mathbf{c}$ is the total of plants unique in the communities.

The Kruskal-Wallis test was used to evaluate whether there were differences in knowledge between men and women and if social-economic factors influenced the species citations [29]. The data were organized as follows: age (1: $\leq 30$ years old; $2: 31 \leq 40$ years old; $3: 41 \leq 50$ years old; 4: $51 \leq 60$ years old; 5 : $\geq 61$ years old), gender (woman; man), family income ( $1: \leq 1$ minimum wage; $2:>1$ minimum wage), and education level (illiterate, for people who have never attended school; basic education, for people who attended only the first years of school; middle school).

\section{Socioeconomic profile of the communities}

In this study, 44 people were interviewed; 27 of them were in the Cachoeira community, including 16 women aged 24 to 92 years old, of whom $56 \%$ were involved in domestic activities and $44 \%$ were salaried employees. The men were aged 26 to 65 years old, with $90 \%$ engaged in agricultural activities and animal breeding and the rest of them (10\%) working in other activities.

With regard to education level, $75 \%$ of the population is illiterate; the rest of them (25\%) have attended school sometime, but none of them have finished high school. The maximum income in the community does not exceed two minimum wages for $45 \%$ of the interviewees, and the other individuals have an income of one minimum wage.

In Barrocas, $70 \%$ of the men (9), aged 31 to 71 years old, are farmers and supplement the family income by breeding animals such as swine, horses, goats, sheep and cattle. Regarding women (8), aged 38 to 73 years old, $33 \%$ are also farmers, $30 \%$ work in several activities for the government and for private employers, and 37\% are engaged in domestic activities.

In this community, $50 \%$ of people live with a minimum wage as an income, and the others earn just a little more than that minimum wage except for a registered case whose informant earns an average of five salaries as retirement. 


\section{Results}

\section{Local knowledge about fodder plants}

The respondents displayed vast knowledge, citing more than 1,600 pieces of information about plants (mean $36 \pm 16.73$ by informant). They identified 136 species that were distributed into 113 genera and 37 families. The species include members of the families Poaceae (13), Fabaceae (13), Euphorbiaceae (8) and Cactaceae (7) (Figure 1), which emphasizes the richness of the species diversity. More than $60 \%$ of the information is related to actual use, with a significant representation (978, mean: $8.15 \pm 7.3$ ) when compared to other known species with potential in animal nutrition $(634$, mean $=$ $5.28 \pm 5.07),[\mathrm{Z}(\mathrm{U})=1.9293 ; \mathrm{p}<0.05]$.

The plants indicated by the local experts came from the participant observations. These people accompany the animal throughout the day and observe its preferences; however, in critical periods of drought, the choice is no longer the animal's but becomes the owner's, who collect and prepare food for the animal. The best example of this is the use of Opuntia ficus-indica (L.) Mill., other cacti such as Pilosocereus gounellei (F. A.C. Weber) Byles \& G.D. Rowley, bromeliads such as Bromelia laciniosa Mart. ex. Schult. f., and the leaves and branches of fruit trees such as Prosopis juliflora (Sw.) DC. These are good choices, given their availability, productivity (produced dry weight ratio) and nutritional value.

There was a high similarity (0.75) in the knowledge of residents of the two communities, as there were 99 plant species mentioned in both. Despite the high similarity and geographic proximity between the communities, some plants were registered in only one community: 19 in Barrocas and 18 in Cachoeira. These species had a low frequency of citation (5\%), and some of them are cultivated in agroforest backyards, such as Ricinus communis L., Mangifera indica L., Moringa oleifera Lam. and Anacardium occidentale L. As shown in Table 1, others corresponded to species of past use, which were usually plants that are no longer found in the region, such as Licania rigida Benth., Parkinsonia aculeata L. and Combretum leprosum Mart.

Finally, for all species, the informants indicated the plant parts preferentially consumed by animals. The parts most frequently consumed were leaves (52.52\%), ranging from trees to herbaceous plants, followed by fruits and cladodes $(12.61 \%$ and $11.45 \%$, respectively) and the entire plant (10.47\%) (Figure 2). Considering the number of plant parts, $20 \%$ were indicated to have only one part of the plant used for food, while the remaining species had more than two parts mentioned as animal feed (Figure 3).

\section{Use of native and exotic plants}

Seventy identified species were classified as exotic and 67 as native. Thus, with regard to their biogeographical origin, there were no significant differences between native and exotic plants $\left(\chi^{2}=0.06, \mathrm{p}>0.05\right)$.

The following native species are highlighted as the most cited: Pilosocereus gounellei (F.A.C. Weber) Byles \& G.D. Rowley (xique-xique, 85), Spondias tuberosa Arruda (umbu, 63), Tacinga palmadora (Britton \& Rose) N.P. Taylor \& Stuppy (Palmatória, 49), Bromelia laciniosa Mart. ex. Schult. f. (macambira, 42), Ziziphus joazeiro Mart. (juazeiro, 40), Cereus jamacaru DC (mandacaru, 39) and Caesalpinia pyramidalis Tul. (catingueira, 36). The most cited exotic species were Amaranthus cruentus L. (bredo de porco, 48), Prosopis juliflora (Sw.) DC. (algaroba, 45), Cenchrus echinatus L. (capim, 39) and Opuntia ficus-indica (L.) Mill. (cactus, 38) (Table 1).

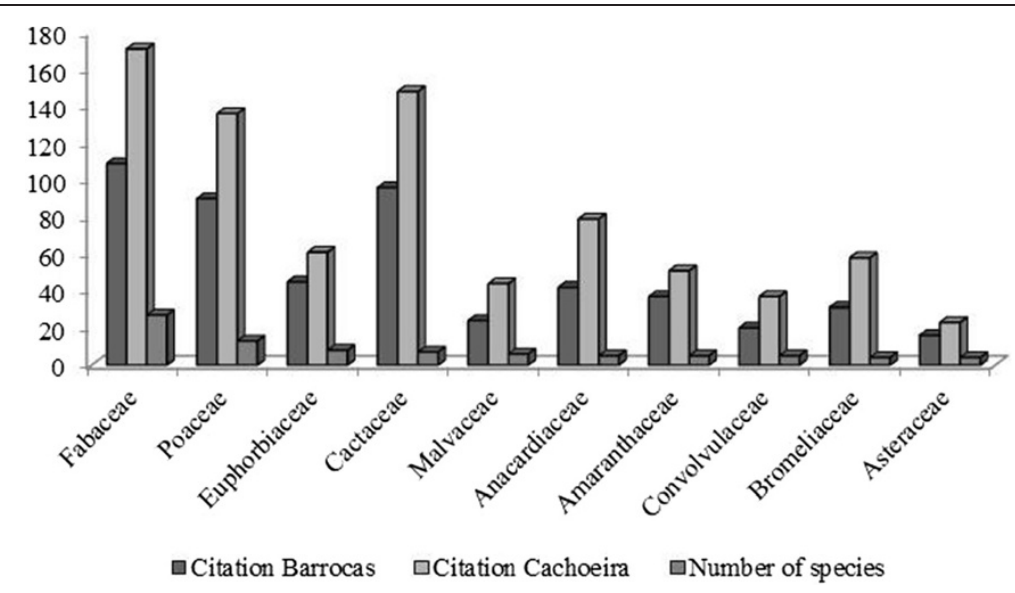

Figure 1 Families represented by the greatest number of fodder species mentioned in the communities of Cachoeira and Barrocas (Soledade, Paraíba, NE Brazil). 
Table 1 Forage plants mentioned by the interviewed in Barrocas (A) and Cachoeiras (B), in Soledade city - PB,NE Brazil

\begin{tabular}{|c|c|c|c|c|c|c|c|c|}
\hline \multirow[t]{2}{*}{ Family } & \multirow[t]{2}{*}{ Species } & \multirow[t]{2}{*}{ Vernacular name } & \multirow[t]{2}{*}{$\begin{array}{l}\text { Part } \\
\text { consumption }\end{array}$} & \multicolumn{2}{|c|}{$\begin{array}{l}\text { Citations per } \\
\text { community }\end{array}$} & \multirow[t]{2}{*}{ Origin } & \multirow[t]{2}{*}{$\begin{array}{l}\text { Life } \\
\text { form }\end{array}$} & \multirow[t]{2}{*}{$\begin{array}{l}\text { Frequency } \\
(\%)\end{array}$} \\
\hline & & & & A & B & & & \\
\hline Agavaceae & Agave sisalana Perrine ex Engler & Agave & $\mathrm{LV}, \mathrm{Tb}$ & 3 & 2 & E & Herb & 32.56 \\
\hline Alismataceae & $\begin{array}{l}\text { Echinodorus andrieuxii (Hook. and Arn.) } \\
\text { Small }\end{array}$ & Golfe & LV & - & 1 & $\mathrm{~N}$ & Herb & 2.33 \\
\hline \multirow[t]{5}{*}{ Amaranthaceae } & Alternanthera tenella Colla & Quebra panela & LV, P.t. & 5 & 8 & $\mathrm{~N}$ & Herb & 34.88 \\
\hline & Amaranthus spinosus L. & Bredo de espinho & LV, P.t. & 7 & 8 & E & Herb & 30.23 \\
\hline & Amaranthus cruentus L. & Bredo de porco & LV, P.t. & 14 & 19 & $\mathrm{E}$ & Herb & 100 \\
\hline & Amaranthus viridis $\mathrm{L}$. & Bredo & LV, P.t. & 5 & 8 & E & Herb & 30.23 \\
\hline & Gomphrena demissa Mart. & Cama de amancebado & P.t. & 1 & - & $\mathrm{N}$ & Herb & 2.33 \\
\hline \multirow[t]{5}{*}{ Anacardiaceae } & Anacardium occidentale L. & Caju & LV & 1 & - & $\mathrm{N}$ & Tree & 2.33 \\
\hline & Mangifera indica L. & Manga & LV & 1 & - & $\mathrm{E}$ & Tree & 2.33 \\
\hline & Myracrodruon urundeuva Allemão & Aroeira & $\mathrm{Lv}, \mathrm{Fr}, \mathrm{Se}, \mathrm{Br}$ & 13 & 18 & $\mathrm{~N}$ & Tree & 72.09 \\
\hline & Schinopsis brasiliensis Engl. & Braúna & $\mathrm{LV}, \mathrm{Fr}, \mathrm{Br}$ & 8 & 17 & $\mathrm{~N}$ & Tree & 58.14 \\
\hline & Spondias tuberosa Arruda & Umbuzeiro & $\mathrm{LV}, \mathrm{Fr}, \mathrm{Br}$ & 19 & 44 & $\mathrm{~N}$ & Tree & 100 \\
\hline \multirow[t]{2}{*}{ Apocynaceae } & Aspidosperma pyrifolium Mart. & Pereiro & $\mathrm{LV}, \mathrm{Fr}, \mathrm{Br}$ & 6 & 17 & N & Tree & 53.49 \\
\hline & Acanthospermum hispidum DC. & Espinho de cigano & LV, P.t. & 3 & 5 & E & Herb & 32.56 \\
\hline \multirow[t]{3}{*}{ Asteraceae } & Blainvillea acmella (L.) Philipson & Bamburrá & LV, P.t. & 6 & 4 & E & Herb & 23.26 \\
\hline & Helianthus annus L. & Girassol & LV & 3 & 8 & E & Herb & 25.58 \\
\hline & Simsia dombeyana DC. & Espinho de agulha & LV, P.t. & 4 & - & $\mathrm{N}$ & Herb & 9.3 \\
\hline \multirow[t]{2}{*}{ Bombacaceae } & Ceiba glaziovii (Kuntze) K. Schum. & Barriguda & LV, Rz & 4 & 10 & N & Tree & 32.56 \\
\hline & $\begin{array}{l}\text { Pseudobombax marginatum (A.St.-Hil.) } \\
\text { A. Robyns }\end{array}$ & Imbiratanha & LV & - & 2 & N & Tree & 4.65 \\
\hline \multirow[t]{5}{*}{ Boraginaceae } & Heliotropium elongatum (Lehm.) I.M. Johnst. & Crista de peru & P.t. & 4 & 2 & $\mathrm{~N}$ & Herb & 13.95 \\
\hline & Heliotropium indicum L. & Fedegoso & LV & 11 & 4 & E & Herb & 34.88 \\
\hline & Heliotropium procumbens Mill. & Mato-azul & P.t. & 4 & 6 & E & Herb & 23.26 \\
\hline & Heliotropium tiaridioides Cham. & Crista de peru & P.t. & 4 & 8 & E & Herb & 27.91 \\
\hline & Varronia leucocephala (Moric.) J.S.Mill. & Maria preta & LV & 1 & 1 & E & Shrub & 4.65 \\
\hline \multirow[t]{4}{*}{ Bromeliaceae } & Bromelia laciniosa Mart. ex. Schult. f. & Macambira rôxa & $\mathrm{LV}, \mathrm{Tb}$ & 17 & 25 & $\mathrm{~N}$ & Herb & 97.67 \\
\hline & Encholirium sp. & Macambira branca & $\mathrm{LV}, \mathrm{Tb}$ & 5 & 17 & $\mathrm{~N}$ & Herb & 48.84 \\
\hline & Neoglaziovia variegata (Arruda) Mez & Caroá & $\mathrm{LV}, \mathrm{Tb}$ & 8 & 17 & $\mathrm{~N}$ & Herb & 58.14 \\
\hline & Tillandsia recurvata (L.) L. & Salambaia & P.t. & 1 & - & E & Herb & 2.33 \\
\hline \multirow[t]{7}{*}{ Cactaceae } & Cereus jamacaru DC. & Cardeiro/mandacaru & Fr, P.t. & 16 & 23 & $\mathrm{~N}$ & Tree & 90.7 \\
\hline & $\begin{array}{l}\text { Melocactus zehntneri (Britton \& Rose) } \\
\text { Luetzelb. }\end{array}$ & Coroa de frade & P.t. & 11 & 15 & $\mathrm{~N}$ & Herb & 60.47 \\
\hline & Opuntia ficus-indica (L.) Mill. & Palma* & $\mathrm{Fr}, \mathrm{Cd}$ & 14 & 24 & E & Shrub & 88.37 \\
\hline & $\begin{array}{l}\text { Pilosocereus gounellei (F.A.C. Weber) Byles \& } \\
\text { G.D. Rowley }\end{array}$ & Xique-xique & $\mathrm{Fr}, \mathrm{Cd}$ & 36 & 49 & $\mathrm{~N}$ & Shrub & 100 \\
\hline & Pilosocereus pachicladus F. Ritter. & Facheiro & $\mathrm{LV}, \mathrm{Cd}$ & 8 & 14 & $\mathrm{~N}$ & Shrub & 86.4 \\
\hline & Tacinga inaenamoema Britton \& Rose & Cumbeba & $\mathrm{Fr}, \mathrm{Cd}$ & 2 & 1 & $\mathrm{~N}$ & Shrub & 6.98 \\
\hline & $\begin{array}{l}\text { Tacinga palmadora (Britton\& Rose) N. P. } \\
\text { Taylor \& Stuppy }\end{array}$ & Palmatória & $\mathrm{Fr}, \mathrm{Cd}$ & 17 & 32 & $\mathrm{~N}$ & Shrub & 100 \\
\hline Capparaceae & Cynophalla flexuosa (L.) J. Presl & Feijão-brabo & $\mathrm{LV}, \mathrm{Fr}, \mathrm{Br}$ & 9 & 15 & $\mathrm{~N}$ & Tree & 55.81 \\
\hline Celastraceae & Maytenus rigida Mart. & Bom Nome & $\mathrm{Lv}, \mathrm{Br}$ & 2 & 2 & $\mathrm{~N}$ & Tree & 9.3 \\
\hline Chrysobalanaceae & Licania rigida Benth. & Oiticica & $\mathrm{Lv}, \mathrm{Fr}$ & - & 1 & $\mathrm{~N}$ & Tree & 2.33 \\
\hline Convolvulaceae & Jacquemontia bahiensis O'Donell & Amarra cachorro & LV & 3 & 11 & $\mathrm{E}$ & Herb & 32.56 \\
\hline
\end{tabular}


Table 1 Forage plants mentioned by the interviewed in Barrocas (A) and Cachoeiras (B), in Soledade city - PB,NE Brazil (Continued)

\begin{tabular}{|c|c|c|c|c|c|c|c|c|}
\hline \multirow[t]{2}{*}{ Combretaceae } & Combretum glaucocarpum Mart. & Canela de ema & LV & - & 1 & $\mathrm{~N}$ & Tree & 2.33 \\
\hline & Combretum leprosum Mart. & Mufumbo & $L v, F r$ & 2 & 1 & E & Tree & 6.98 \\
\hline \multirow[t]{2}{*}{ Commelinaceae } & Commelina erecta $\mathrm{L}$. & Olho de Santa Luzia & LV, P.t. & 2 & - & N & Herb & 4.65 \\
\hline & Commiphora leptophloeos (Mart.) J.B. Gillett & Amburana/Imburana & $L V$, & 3 & 12 & E & Tree & 34.88 \\
\hline \multirow[t]{7}{*}{ Convolvulaceae } & Evolvulus glomeratus Nees \& C. Mart. & Flor azul & Lv, Fr & 2 & - & E & Herb & 4.65 \\
\hline & Ipomoea batatas (L.) Lam. & Batata & Lv & - & 1 & $\mathrm{~N}$ & Herb & 2.33 \\
\hline & Ipomoe nil (L.) Roth & Jitirana a & LV, P.t. & 6 & 8 & $\mathrm{~N}$ & Herb & 32.56 \\
\hline & Ipomoea sp. 1 & Jitirana c & LV & 5 & 8 & - & Herb & 30.23 \\
\hline & Jacquemontia hirsuta Choisy & Jitirana & LV, P.t. & 8 & 14 & & Herb & 32.56 \\
\hline & Merremia aegyptia (L.) Urb. & Jitirana (branca) & LV, P.t. & 17 & 3 & $E$ & Herb & 46.51 \\
\hline & Merremia sp. 2 & Jitirana & P.t. & 3 & 5 & E & Herb & 18.6 \\
\hline \multirow[t]{4}{*}{ Cucurbitaceae } & Cucumis anguria $\mathrm{L}$. & Maxixe* & LV & 1 & - & E & Herb & 2.33 \\
\hline & Cucurbita sp. & Jerimum* & LV & 3 & 2 & E & Herb & 11.63 \\
\hline & Cucurbita sp. & Pepino* & LV & 1 & 1 & E & Herb & 4.65 \\
\hline & Momordica charantia L. & Melão de São Caetano* & Lv, Fr & 1 & - & E & Herb & 2.33 \\
\hline \multirow[t]{2}{*}{ Cyperaceae } & Cyperus uncinulatus Schrad. ex Nees & Barba de bode & Lv, Fr, P.t. & 6 & 4 & E & Herb & 23.26 \\
\hline & Citrullus lanatus (Thumb.) Matsum. \& Nakai & Melancia* & LV & 3 & 2 & $E$ & Herb & 11.63 \\
\hline \multirow[t]{6}{*}{ Euphorbiaceae } & Cnidoscolus quercifolius Pohl & Favela & LV & - & 1 & N & Tree & 2.33 \\
\hline & Croton blanchetianus Baill. & Marmeleiro & LV, Fr., Br & 16 & 20 & $\mathrm{~N}$ & Tree & 83.72 \\
\hline & Croton heliotropiifolius Kunth. & Quebra-faca & LV & 1 & 2 & E & Tree & 6.98 \\
\hline & Croton sincorensis Mart. & Marmeleiro branco & LV & - & 1 & $\mathrm{~N}$ & Tree & 2.33 \\
\hline & Euphorbia tirucalli L. & Aveloz & LV & 9 & 8 & E & Tree & 39.53 \\
\hline & Ricinus communis $\mathrm{L}$. & Carrapateira & LV & 1 & - & E & Tree & 2.33 \\
\hline \multirow[t]{20}{*}{ Fabaceae } & Amburana cearensis (Allemão) A.C.Sm. & Cumaru & $\mathrm{Lv}, \mathrm{Fr}, \mathrm{Br}$ & - & 2 & $\mathrm{~N}$ & Tree & 4.65 \\
\hline & Anadenanthera colubrina (Vell.) Brenan & Angico & $\mathrm{LV}, \mathrm{Fr}, \mathrm{Br}$ & 3 & 7 & N & Tree & 23.26 \\
\hline & Bauhinia cheilantha (Bong.) Steud. & Mororó & $\mathrm{LV}, \mathrm{Fr}, \mathrm{Br}$ & 3 & 5 & $\mathrm{~N}$ & Tree & 18.6 \\
\hline & Calliandra sp. & Pimenta d'água & P.t. & 1 & 1 & - & Herb & 4.65 \\
\hline & Chamaecrista rotundifolia (Pers.) Greene. & Malícia & LV & - & 2 & $\mathrm{~N}$ & Herb & 4.65 \\
\hline & Capparis hastata Jacq. & Feijão de boi & LV & 1 & - & N & Tree & 2,33 \\
\hline & Chloroleucon mangense (Jacq.) Britton \& Rose & Coronha braba & $\mathrm{Fr}$ & - & 1 & N & Tree & 2.33 \\
\hline & Crotalaria incana L. & Chocalho de raposa & LV & 1 & 2 & $\mathrm{~N}$ & Herb & 6.98 \\
\hline & Desmodium glabrum (Mill.) DC. & Feijão de rolinha & LV, P.t. & 3 & 5 & N & Shrub & 23.26 \\
\hline & Desmodium distortum (Aubl.) J.F. Macbr. & Rapadura de cavalo & LV, P.t. & 5 & 16 & N & Shrub & 48.84 \\
\hline & Erythrina velutina Willd. & Mulungu & Lv, Fr, Se & 3 & 5 & $\mathrm{~N}$ & Tree & 18.6 \\
\hline & $\begin{array}{l}\text { Froelichia humboldtiana (Roem. \& Schult.) } \\
\text { Seub. }\end{array}$ & Ervanço & LV, P.t. & 5 & 6 & N & Herb & 25.58 \\
\hline & Gliricidia sepium (Jacq.) Kunth ex Walp. & Gliricídia & $\mathrm{Lv}, \mathrm{Fr}, \mathrm{Br}$ & 1 & 1 & E & Tree & 4.65 \\
\hline & Glycine max(L.) Merr. & Soja* & - & - & 1 & E & Herb & 2.33 \\
\hline & Indigofera suffruticosa Mill. & Anil & LV & 4 & 2 & E & Herb & 13.95 \\
\hline & Inga sp. & Ingazeira & LV, Fr, Se & 4 & 3 & - & Tree & 16.27 \\
\hline & Leucaena leucocephala (Lam.) Dewit & Leucena & LV & 2 & 5 & N & Shrub & 16.27 \\
\hline & Libidibia ferrea (Mart. ex Tul.) L.P. Queiroz & Jucá & $\mathrm{Lv}, \mathrm{Fr}, \mathrm{Br}$ & 2 & 3 & N & Tree & 11.63 \\
\hline & Mimosa ophthalmocentra Mart. ex. Benth. & Jurema de imbira & $\mathrm{LV}, \mathrm{Fr}, \mathrm{Br}$ & 6 & 8 & E & Tree & 32.56 \\
\hline & Mimosa tenuiflora (Willd.) Poir. & Jurema-preta & LV & 12 & 12 & $\mathrm{~N}$ & Tree & 55.81 \\
\hline
\end{tabular}


Table 1 Forage plants mentioned by the interviewed in Barrocas (A) and Cachoeiras (B), in Soledade city - PB,NE Brazil (Continued)

\begin{tabular}{|c|c|c|c|c|c|c|c|c|}
\hline & Parkinsonia aculeata $\mathrm{L}$. & Turco & P.t. & 2 & 1 & $\mathrm{~N}$ & Tree & 6.97 \\
\hline & Phaseolus vulgaris $\mathrm{L}$. & Feijão* & P.t. & 10 & 22 & $\mathrm{~N}$ & Herb & 74.42 \\
\hline & Piptadenia stipulacea (Benth.) Ducke & Jurema branca & P.t. & 12 & 8 & $\mathrm{~N}$ & Tree & 46.51 \\
\hline & Poincianella pyramidalis Tul. & Catingueira & $\mathrm{LV}, \mathrm{Fr}, \mathrm{Br}$ & 11 & 23 & $\mathrm{~N}$ & Tree & 79.07 \\
\hline & Prosopis juliflora (Sw.) DC. & Algaroba & $\mathrm{Fr}, \mathrm{Br}$ & 17 & 27 & $E$ & Tree & 100 \\
\hline & Senna obtusifolia (L.) H.S. Irwin \& Barneby & Fedegoso & $\mathrm{Lv}, \mathrm{Br}$ & 2 & 2 & $\mathrm{~N}$ & Shrub & 9.3 \\
\hline & Rhynchosia minima DG. & Feijão de rolinha & LV & 1 & 3 & $\mathrm{~N}$ & Shrub & 9.3 \\
\hline & $\begin{array}{l}\text { Vigna peduncularis var. peduncularis (Kunth) } \\
\text { Fawc. \& Rendle }\end{array}$ & Feijão miúdo & LV & 1 & 1 & $\mathrm{~N}$ & Herb & 4.65 \\
\hline Loasaceae & Mentzelia aspera L. & Amor de velho & $\mathrm{Br}$ & 1 & 1 & $\mathrm{~N}$ & Herb & 4.65 \\
\hline Lythraceae & Pleurophora anomala (A.St.-Hill.)Koehne & Vassourinha & $\mathrm{Br}$ & 1 & - & $E$ & Herb & 2.33 \\
\hline \multirow[t]{8}{*}{ Malvaceae } & Gossypium hirsutum L. & Algodão* & $\mathrm{Lv}, \mathrm{Fr}$ & 2 & 9 & E & Shrub & 25.58 \\
\hline & Herissantia crispa (L.) Brizicky & Mela bode & P.t. & 4 & 5 & $\mathrm{~N}$ & Shrub & 20.93 \\
\hline & Manihot dichotoma Ule & Maniçoba & $\mathrm{LV}, \mathrm{Fr}$ & 8 & 15 & $\mathrm{~N}$ & Tree & 53.49 \\
\hline & Melochia tomentosa L. & Malva rôxa & LV, P.t. & 4 & - & E & Herb & 9.3 \\
\hline & Pavonia cancellata Cav. & Jitirana de boi & LV & 1 & - & $\mathrm{N}$ & Herb & 2.33 \\
\hline & Sida acuta Burm. f. & Malva relógio & LV, P.t. & 4 & 2 & N & Herb & 13.95 \\
\hline & Sida galheirensis Ulbr. & Malva amarela & P.t. & 17 & 24 & $\mathrm{~N}$ & Shrub & 95.35 \\
\hline & Sida rombifolia $\mathrm{L}$. & Malva-preta & P.t. & - & 1 & E & Shrub & 2.33 \\
\hline Moringaceae & Moringa oleifera Lam. & Muringa & Lv, Fr, P.t. & 2 & - & E & Tree & 4.65 \\
\hline Myrtaceae & Psidium guajava L. & Goiaba & P.t. & 1 & - & $\mathrm{N}$ & Tree & 2.33 \\
\hline Nyctaginaceae & Boerhavia difussa L. & Pega-pinto & P.t. & 8 & 13 & $E$ & Herb & 27.91 \\
\hline Plantaginaceae & Stemodia sp. & Meladinha & LV & 1 & - & E & Herb & 2.33 \\
\hline \multirow[t]{16}{*}{ Poaceae } & Anthephora hermaphrodita (L.) Kuntze & $\begin{array}{l}\text { Capim comum/capim } \\
\text { native }\end{array}$ & LV & 12 & 15 & E & Herb & 62.79 \\
\hline & Brachiaria decumbens Stapf & Capim braquiária* & LV & 3 & 9 & E & Herb & 27.91 \\
\hline & Cenchrus brownii Roem. \& Schult. & Carrapicho de cavalo & LV, P.t. & 4 & 2 & E & Herb & 13.95 \\
\hline & Cenchrus ciliares L. & Capim-buffel & LV & 3 & 3 & E & Herb & 13.95 \\
\hline & Cenchrus echinatus L. & Capim-carrapicho & LV & 15 & 24 & E & Herb & 90.7 \\
\hline & Chloris barbata Sw. & Capim pé-de-galinha & LV & 5 & 8 & E & Herb & 30.23 \\
\hline & Chloris orthonoton Doll. & Capim-de raiz & LV & 6 & 6 & E & Herb & 27.91 \\
\hline & Dactyloctenium aegyptium (L.) Willd. & Capim pé-de-galinha & LV, P.t. & 3 & 12 & E & Herb & 34.88 \\
\hline & Enteropogon mollis (Nees) Clayton & Capim do mato & LV & 12 & 5 & $\mathrm{~N}$ & Herb & 39.53 \\
\hline & Leersia hexandra Sw. & Capim marreco & LV & 8 & 4 & $\mathrm{~N}$ & Herb & 27.91 \\
\hline & Pennisetum purpureum Schum & Capim-elefante* & LV & 11 & 13 & E & Herb & 55.81 \\
\hline & Tragus berteronianus Schult. & Carrapicho de ovelha & LV & 6 & 10 & E & Herb & 37.21 \\
\hline & Saccharum sp. & Cana-de-açúcar* & LV & 2 & 2 & E & Herb & 9.3 \\
\hline & Sorghum bicolor (L.) Moench & Milho sorgo* & LV & 6 & 15 & E & Herb & 48.84 \\
\hline & Triticum sp. & Trigo* & LV & 1 & - & E & Herb & 2.33 \\
\hline & Zea mays L. & Milho* & LV & 14 & 22 & E & Herb & 83.72 \\
\hline \multirow[t]{3}{*}{ Portulacaceae } & Portulaca elatior Mart. ex. Rohrb. & Bredo de alecrim & LV & 2 & - & E & Herb & 4.65 \\
\hline & Portulaca halimoides $\mathrm{L}$. & Alecrim do mato & LV, P.t. & 1 & - & E & Herb & 2.33 \\
\hline & Portulaca oleracea L. & Berdruega/Bredo gordo & LV, P.t. & 4 & 3 & E & Herb & 16.28 \\
\hline Rhamnaceae & Zizyphus joazeiro Mart. & Juá & $\mathrm{Lv}, \mathrm{Fr}, \mathrm{Br}$ & 14 & 26 & $\mathrm{~N}$ & Tree & 93.02 \\
\hline
\end{tabular}


Table 1 Forage plants mentioned by the interviewed in Barrocas (A) and Cachoeiras (B), in Soledade city - PB,NE Brazil (Continued)

\begin{tabular}{|c|c|c|c|c|c|c|c|c|}
\hline \multirow[t]{3}{*}{ Rubiaceae } & Borreria verticillata (L.) G. Mey. & Vassourinha de botão & LV & - & 1 & E & Herb & 2.33 \\
\hline & Diodia teres Walter & Mata pasto & LV & 0 & 2 & E & Herb & 4.65 \\
\hline & Richardia grandiflora (Cham. \& Schltdl.) Steud. & Melancia de vaca & $\mathrm{LV}, \mathrm{Br}$ & 11 & 13 & E & Herb & 55.81 \\
\hline \multirow[t]{2}{*}{ Sapindaceae } & Cardiospermum oliveirae Ferrucci & Pratudo & Lv, Fr, P.t & 3 & - & E & Herb & 6.98 \\
\hline & Serjania glabrata Kunth. & Mata cachorro & LV & - & 2 & $\mathrm{~N}$ & Herb & 4.65 \\
\hline Sapotaceae & $\begin{array}{l}\text { Sideroxylon obtusifolium (Humb. ex Roem. \& } \\
\text { Schult.) T.D. Penn. }\end{array}$ & Quixabeira & $\mathrm{LV}, \mathrm{Fr}, \mathrm{Br}$ & 14 & 22 & $\mathrm{~N}$ & Tree & 83.72 \\
\hline Scrophulariaceae & Scoparia dulcis L. & Vassourinha & P.t. & 4 & 1 & E & Herb & 11.63 \\
\hline \multirow[t]{3}{*}{ Solanaceae } & Nicotiana glauca R. Graham. & Oliveira & $\mathrm{LV}, \mathrm{Fr}$ & 1 & 1 & E & Shrub & 4.65 \\
\hline & Solanum agrarium Sendtn & Gogoia & LV, Fr & - & 1 & E & Herb & 2.33 \\
\hline & Solanum americanum Mill. & Erva Moura & LV, Fr & 2 & 4 & $\mathrm{~N}$ & Herb & 13.95 \\
\hline Sterculiaceae & Waltheria rotundifolia Schrank & Malva branca & LV, P.t. & 13 & 20 & E & Herb & 76.74 \\
\hline Verbenaceae & Stachytarpheta angustifolia (Mill.) Vahl & Milho de cobra & P.t. & 2 & 1 & $\mathrm{E}$ & Herb & 6.98 \\
\hline
\end{tabular}

Legend. Plant part consumed: $\mathrm{Cl}=$ Cladode; $\mathrm{Fl}=$ flower; $\mathrm{Fr}=$ Fruits; $\mathrm{Br}=$ Branch; $\mathrm{Lv}=$ leaves; P.t. = Entire plant; $\mathrm{Rz}=\mathrm{Rhizome} ; \mathrm{Se}=\mathrm{Seed} ; \mathrm{Status}$ : $\mathrm{N}=\mathrm{Native}$ E = Exotic; Freq. $=$ Frequency of citation

*cultivated.

It was observed that among the plants native and exotic, there was a strong correlation with the period of the year $\left(x^{2}=28.104, p<0.0001\right)$; in other words, during the drought season, there were 44 native species and 13 exotic cited, but during the rainy season, there were 25 native plants and 55 exotic mentioned, meaning that the diet of animals can be conditioned by climatic factors (Figure 4). For example, in the rainy season, there were more citations for herbaceous species represented by species of the Poaceae and Fabaceae families. In the dry season, the highlights were the cactus species, such as $P$. gounellei, and some bromeliads, such as B. laciniosa, both present year round but limited in the dry season.

The most representative families were Poaceae (43\%), Malvaceae (10\%) and Euphorbiaceae (5\%). The most highlighted plants were Waltheria rotundifolia Schrank,
Cenchrus echinatus L., Dactyloctenium aegyptium (L.) Willd., Enteropogon mollis (Nees) Clayton, Blainvillea acmella (L.) Philipson, Froelichia humboldtiana (Roem. \& Schult.) Seub., Jacquemontia hirsuta Choisy and Merremia aegyptia (L.) Urb. In addition, the herbaceous plants in the diet of the animals are complemented during the rainy season by the new leaves of woody plants (Figure 5), beyond the fruit, flowers and inflorescence.

\section{Socioeconomic factors}

At the studied communities, the socioeconomic factors, such as age $(P>0.05)$, income $(P>0.05)$ and education level $(\mathrm{P}>0.05)$, had no influence on the knowledge of fodder plants, except regarding gender, where men have much more knowledge than women. For men, the average number of known plants was $43( \pm 10.67)$ for a total

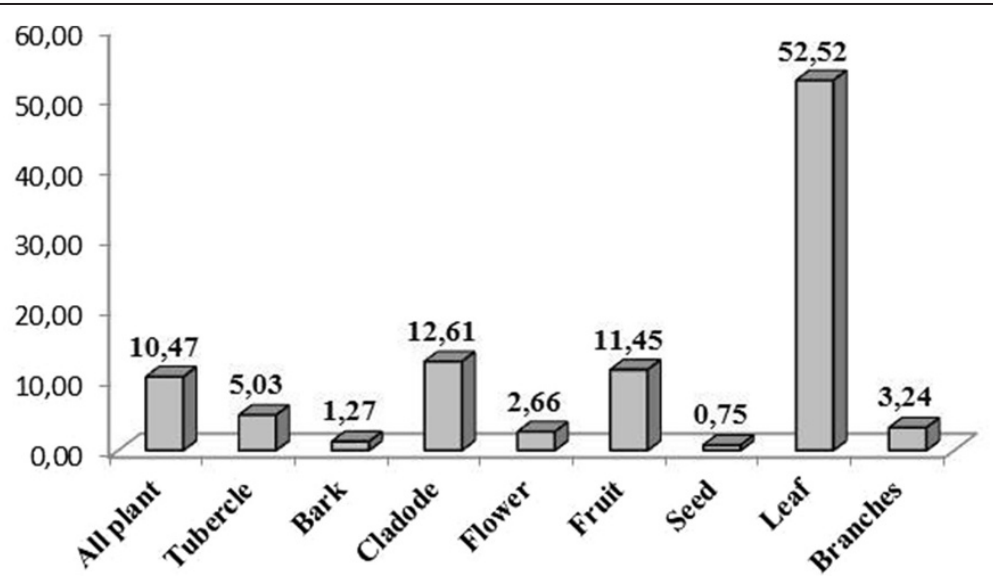

Figure 2 Percentage of citations of plant parts listed in the diet of ruminants of fodder species mentioned in the Cachoeira and Barrocas communities (Soledade, Paraíba, NE Brazil). 


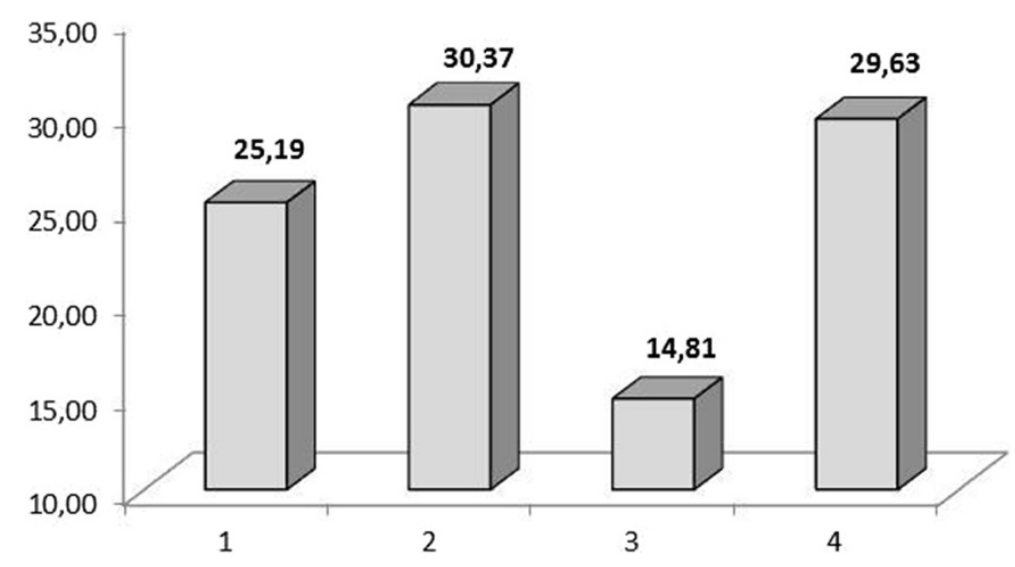

Figure 3 Percentage of citations of plant parts listed in the diet of ruminants in the Cachoeira and Barrocas communities (Soledade, Paraíba, NE Brazil). The numerals represent the number of plant parts used.

of 926 citations, while for women, there were a total of 647 citations, for an average of $26( \pm 10.55)$. The gender difference was statistically significant $(H=11.61$, $\mathrm{p}=0.0007)$.

However, it was observed that in Barrocas, the knowledge between men and women $(\mathrm{H}=1.33, \mathrm{p}>0.05)$ did not differ significantly. In other words, in Barrocas, both men and women know and use these resources, while in Cachoeira, the data demonstrated greater knowledge on the part of the men in relation to women $(\mathrm{H}=14, \mathrm{p}<0.05)$.

\section{Discussion}

The results of this research show that there is a wealth of information for feeding ruminants, with respect to the vegetation composition, nutritional value, palatability and the seasonal availability of the species in both communities, which is a finding similar to those of studies in other countries $(11,14,20)$. These characteristics, palatability, nutritional value and dry matter productivity, are classified by the community as "sweet plants", "fattening plants" and plants for "fill", respectively, and this rating reflects the perceptions of local experts who deal with animals daily. Although this is not a sophisticated classification, as found by Chettri and Sharma (2009) in India, it signals the abundant knowledge of animal food resources of the participants.

\section{Local knowledge of forage plants}

The informants told the interviewers about a higher diversity of useful plants in the feeding of ruminants, compared to prior lists of fodder plants of the Caatinga [3,5,12,30-33]. Those lists show that the Caatinga has a plant diversity that is relatively similar to the plants used by traditional communities in Africa and Mexico [7,8,11,14].

Among the families most often cited, the grasses and Fabaceae are the most prominent in the literature, and

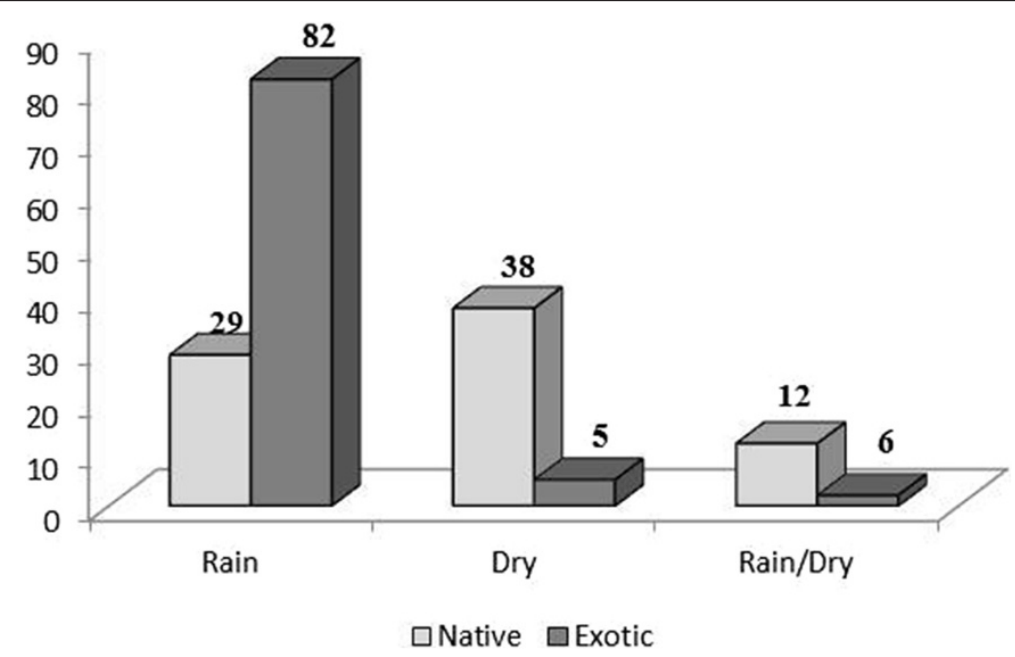

Figure 4 Number of native and exotic plants used in dry and rainy season, in the communities of Barrocas and Cachoeira (Soledade, Paraíba, NE Brazil). 


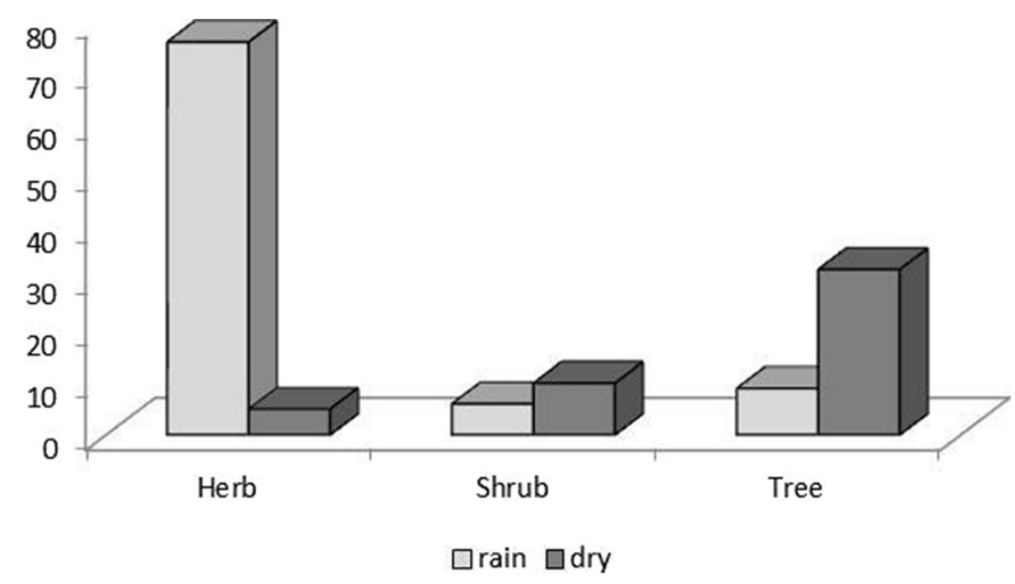

Figure 5 Diversity of species cited in dry and rainy season according to the plant habit in the communities of Barrocas and Cachoeira (Soledade, Paraiba, NE Brazil).

information regarding the potential productivity and nutritional value is abundant, mainly due to the preference of animals for these families (Table 1). The Fabaceae are classified as sweet and fattening plants, (palatable and nutritious) and Poaceae families are classified as palatable and productive, which are highlighted in citations for higher species richness, and are characterized as having high forage potential in Brazilian semiarid areas $[30,32,34,35]$ and in other regions $[8,9,36,37]$.

Locally, the high similarity of local knowledge of fodder resources is a function of the proximity of the communities. On the other hand, the exclusive plants in each community may be reflections of the structural differences of the areas. Groups of species can be common between neighboring areas [7]. For example, Okoli et al. [7] found in three communities of Nigeria that the rearing system could influence the choice of species at each site, i.e., families who kept confined animals demonstrated a greater knowledge about the resources compared with families who raised animals loose in the pasture. However, further investigation is needed to assess the relationship between uses in neighboring areas.

The plant part used in animal feed is an important criterion of the nutritional $[38,39]$ and ecological [40] point of view. In the case of Caatinga, this richness reflects the local diversity and the high variety of resources involved in the diet [3]. The results may reflect the general nature of ruminants adapted to the region that feed from seedling leaves and tree twigs, a fact which confirms prior research in the region $[3,12,30]$. In other regions, the high abundance of resources for animals has been used to understand and to validate the quality of these resources. Investigations carried out in rural Indian communities have disclosed the preference of the informants for woody resources, especially in places with semi-arid climates $[7,11,14,41]$. In some cases, following ethnobotanical inquiries, other species have become listed for nutritional characterization, reinforcing the importance of the local knowledge in the characterization of fodder resources [11,20,41].

Recent research carried out in traditional communities in underdeveloped countries discloses a scenario similar to that found in this study regarding the high dependence on fodder plants in the maintenance of subsistence livestock $[7,9,11,14,15]$, as well as emphasizing the importance of local knowledge as a link in the process of the selection of potential plants for a program of sustainable management and conservation of biodiversity [20].

The present study expands the diversity of species not yet shown in the general listings of forage plants from Northeastern Brazil, with the implications considered as positives for a developed approach, contributing to registering plants and to the awareness of potential species that may be investigated in future research under nutrition, biotechnology and ecological.

\section{Use of native and exotic plants in ruminant feed}

In relation to the biogeographical origin of species, most research in semiarid environments reveals a high diversity of exotic plants in the diet of ruminants, whether spontaneous or cultivated in the communities studied. It is common to note Prosopis juliflora (Sw.) DC., Opuntia ficus-indica (L.) Mill., Brachiaria decumbens Stapf and Chloris orthonoton Doll. due to their importance in production and nutrition $[13,42,43]$.

Native plants are a valuable resource in the communities, as indicated by respondents. These species are distinguished by the availability and nutritional quality to meet the demands of providing animal weight gain and increase in milk production. Further research is needed for these species. 
In the rainy season, the herbaceous plants stand out because they are the most abundant resources, represented mainly by exotic species such as Brachiaria decumbens Stapf and Cenchrus ciliaris L., Merremia aegyptia (L.) Urb. [6]. As rainfall decreases, the herbaceous plants are replaced by the woody species of the region.

Both native and exotic species constitute important elements in the studied communities. Some cultivated species, such as Prosopis juliflora (sw.) DC., Opuntia ficus-indica (L.) Mill., Brachiaria decumbens Stapf and Chloris orthonoton Doll., have been inserted in the region through governmental incentives; these plants are important regarding their productive and nutritional aspects and, with pastures, increase the diversity of the available resources in the diet of the animals [42].

Native plants constitute a valuable resource, according to the indications of the informants. These species are highlighted because of their availability and nutritional quality for meeting animals' requirements, providing weight gain and an increase in milk production. In addition to the benefits of these plants, further study should take into consideration the ecological pressures on these species, which deserve greater attention with regard to management issues and sustainability.

\section{Socioeconomic factors}

Local knowledge may be influenced by variables such as gender, income, age and education level. There is a clear differentiation between men and women; this can be associated with the division of existing work in the communities. Women generally manage feeding and family care, and therefore address medicinal, mystic-religious and ornamental plants [44-47]. In the case of the use of fodder, this relation may vary. For example, men dominated the knowledge about fodder plants in the studied communities, suggesting that they are responsible for animal breeding and the collection of plants. A different result is observed in Pakistan [15], where women and children participate in the collection of forage plants and dominate the resources. These variations are important and must be considered in research involving local communities.

\section{Competing interests}

The authors declare that they have no competing interests.

\section{Authors' contributions}

ATN involved in the study design, conducting of interview, field and laboratory work, and general data collection. UPA main coordinator-supervisor of the research project; contributed also to the study design and data interpretation. RFPL and MVFS contributed to designing and following progress of the research and fieldwork and data analyses. All authors wrote, read and approved the final manuscript.

\section{Acknowledgments}

Thank all informants' communities for participation in research and the reception, especially Maria José de Araújo, Marisete Ramos and Inácio Ramos Vieira. We also thank the Pernambuco State Foundation for Science and Technology (FACEPE) for granting a Doctoral scholarship to the first author, and to the Brazilian National Council of Scientific and Technological Development (Conselho Nacional de Desenvolvimento Científico e Tecnológico - CNPq) for the productivity grants and financial support provided to UPA and MVFS.

Author details

'Laboratório de Etnobiologia Aplicada e Teórica (LEA), Departamento de Biologia da Universidade Federal Rural de Pernambuco, Recife, Pernambuco CEP: 52171-900, Brazil. ²Universidade Federal da Paraíba. Campus II. Centro de Ciências Agrárias, Departamento de Fitotecnia e Ciências Ambientais, Setor de Ecologia e Biodiversidade, Laboratório de Etnoecologia, Areia, Paraíba CEP: 58.397-000, Brazil. ³ Departamento de Zootecnia, Universidade Federal Rural de Pernambuco, Recife, Pernambuco CEP: 52171-900, Brazil.

Received: 23 June 2014 Accepted: 29 December 2014

Published: 10 February 2015

\section{References}

1. FAO. State of the World's Forests. Food and Agriculture Organization. Rome, Italy: 2011. http://www.fao.org.

2. IBGE. Instituto Brasileiro de Geografia e Estatística. Mapa de Biomas e de Vegetação; 2005. www.ibge.gov.br.

3. Maia GN. Caatinga: árvores e arbustos e suas utilidades. São Paulo: Dandz. Computação Gráfica e Editora; 2004.

4. Santos MVF, Lira MA, Dubeux Junior JCB, Guim A, Mello ACL, Cunha MV. Potential of Caatinga forage plants in ruminant feeding. Revista Brasileira de Zootecnia. 2010:39:204-15.

5. Ydoyaga-Santana DF, Lira MA, Santos MVF. Caracterização da caatinga e da dieta de novilhos fistulados, na época chuvosa, no semiárido de Pernambuco. Revista Brasileira de Zootecnia. 2011;40(1):69-78

6. Filho JMP, Bakke OA. Produção de forragem de espécies herbáceas da caatinga. In: Uso sustentável e conservação dos recursos florestais da caatinga. Gariglio: Maria Auxiliadora. Brasília: Serviço Florestal Brasileiro; 2010

7. Okoli IC, Ebere CS, Uchegbu MC, Udah CA, Ibeawuchi II. A survey of the diversity of plants utilized for small ruminant feeding in south-eastern Nigeria. Agriculture, Ecosystems and Environment. 2003;96:147-54

8. Awan AA, Akhtar T, Dar MEUI. Fodder plants of some selected areas of Jhelum valley district Muzaffarabad Azad Kashmir. Pak J Biol Sci. 2007;10:1547-9.

9. Tabuti JRS, Lye KA. Fodder Plants for Cattle in Kaliro District, Uganda. Afr Stud Monogr. 2009;3:161-70

10. Meissner HH. Recent research on forage utilization livestock in South Africa by ruminant. Animal Feed Science Technology. 1997;69:103-19.

11. Nahed J, Villafuerte AL, Grande AD, Perez-Gil BF, Alemh T, Carmona AJ. Fodder shrub and tree species in the Highlands of southern Mexico. Animal Feed Science Technology. 1997;68:213-23

12. Araújo Filho JA, Carvalho FC, Gadelha JA, Leite ER. Composição botânica e química da dieta de ovinos e caprinos em pastoreio combinado na região Inhamuns, Ceará. Revista Brasileira de Zootecnia. 1996;25:383-95.

13. Araújo Filho JA, Silva NL. Manipulação da Vegetação da Caatinga para Produção Sustentável de Forragem. 2011. http://faeb.org.br/detalhefaeb.html? tx_ttnews\%5Btt_news\%5D=813\&cHash=44a9bd2311e029ce6d7ab9ba6fcd6527.

14. Thapa $A B$, Walker $D H$. Sinclair'Indigenous knowledge of the feeding value of tree fodder. Animal Feed Science Technology. 1997;68:37-54.

15. Aumeeruddy-Thomas AYA, Shinwari ZK, Ayaz A, Khan AA. Ethnobotany and management of fodder and fuelwood at Ayubia National Park, North West Frontier Province. WWF. Godalming UK: Pakistan. People and Plants Working Paper; 2004

16. Ferraz JSF, Meunier IMJ, Albuquerque UP. Con hecimento sobre espécies lenhosas úteis da mata ciliar do Riacho do Navio, Floresta, Pernambuco. Zonas Áridas. 2005:9:27-39

17. Lucena RFP, Nascimento VT, Araúijo EL, Albuquerque UP. Local Uses of Native Plants in an Area of Caatinga Vegetation (Pernambuco, NE Brazil). Ethnobotany Research and Applications. 2008;6:003-13.

18. Santos LL, Ramos MA, Silva SI, Sales MF, Albuquerque UP. Caatinga Ethnobotany: Anthropogenic Landscape Modification and Useful Species in Brazil's Semi-Arid Northeast. Econ Bot. 2009;63:363-74. 
19. Papanastasisa VP, Platisb PD, Dini-Papanastasi O. Effects of age and frequency of cutting on productivity of Mediterranean deciduous fodder tree and shrub plantations. For Ecol Manage. 1998;110:283-92.

20. Chettri N, Sharma E. A scientific assessment of traditional knowledge on firewood and fodder values in Sikkim. India For Ecol Manage. 2009:257:2073-8

21. Ramos MA, Medeiros PM, Almeida ALS, Feliciano ALP, Albuquerque UP. Use and knowledge of fuelwood in an area of Caatinga Vegetation in NE Brazil. Biomass and Bioenergy. 2008;32:510-7.

22. Almeida CFCBR, Ramos MA, Amorim ELC, Albuquerque UP. A comparison of knowledge about medicinal plants for three rural communities in the semi-arid region of northeast of Brazil. J Ethnopharmacol. 2010;127:674-84.

23. Albuquerque UP, Lucena RFP, Alencar NL. Methods and Techniques Used to Collect Ethnobiological Data. In: Albuquerque U, Cruz da Cunha L, Lucena R, Alves R, editors. Methods and techniques in Ethnobiology and Ethnoecology. New York: Springer; 2014. p. 15-37.

24. BRASIL. Ministério da Integração Nacional. Brasília: MIN. Nova delimitação do semi-árido brasileiro; 2006.

25. CPRM - Serviço Geológico do Brasil. Projeto cadastro de fontes de abastecimento por água subterrânea. In: Mascarenhas JC, Beltrão BA, Souza Junior LC, Morais F, Mendes VA, Miranda JLF, editors. Diagnóstico do município de Gurjão, Estado da Paraíba. Recife: CPRM/PRODEEM 10; 2005.

26. Sá IMM, Marangon LC, Hanazaki N, Albuquerque UP. Use and knowledge of fuelwood in three rural Caatinga (dryland) communities in NE Brazil. Environ Dev Sustain. 2009;11:833-52.

27. Ayres MJR, Ayres DL, Santos AS. Aplicações estatísticas nas áreas das ciências biológicas e médicas. Belém, Pará, Brasil: BioEstat (5.0); 2007.

28. Forzza RC, Leitman PM, Costa A, Carvalho Jr AA. Catálogo de plantas e fungos do Brasil. Vols. 1 e 2. Rio de Janeiro, Brazil: Instituto de Pesquisas Jardim Botânico do Rio de Janeiro; 2010.

29. Sokal RR, Rholf FG. Biometry. New York: Freeman and Company; 1995.

30. Nascimento MPSC, Oliveira MEA, Carvalho JH. Nascimento HTS. Forrageiras da Bacia do Parnaíba: usos e composição química. Terezina: EMBRAPACPAMN/Recife. Associação Plantas do Nordeste; 1996. p. 86.

31. Lima JLS. Plantas Forrageiras da Caatinga - Usos e potencialidades. Petrolina-PE: EMBRAPA/CPATSA/PNE/RBG-KEW; 1996. p. 44.

32. Sampaio EVSB, Rodal MJ. Usos das plantas da caatinga. In: Sampaio EVSB, Giulietti AM, Virgínio J, Gamarra-Rojas CFL, editors. Vegetação e flora da caatinga. Recife, Brazil: APNE - CNIP; 2002. p. 176-90.

33. Vieira EL, Carvalho FFR, Batista AMV, Ferreira RLC, Santos MVF, Lira MA, et al. Composição química de forrageiras e seletividade de bovinos em bosque de Sabiá (Mimosa caesalpiniifolia Benth.) nos períodos chuvosos e secos. Revista Brasileira de Zootecnia. 2005;34(5):1505-11.

34. Araújo Filho JA. Manipulação da vegetação lenhosa da caatinga para fins pastoris. Sobral, CE: Empresa Brasileira de Pesquisa Agropecuária. (EMBRAPA-CNPC. Circular Técnica, 11); 1992.

35. Costa JAS, Nunes TS, Ferreira APL, Stradmann MTS, Queiroz LP. Leguminosas forrageiras da Caatinga: espécies importantes para as comunidades rurais do sertão da Bahia. Feira de Santana: Universidade Estadual de Feira de Santana, SASOP; 2002.

36. Osuji PO, Odenyo AA. The role of legume forages as supplements to low quality roughages - ILRI experience. Animal Feed Science Technology. 1997;69:27-37.

37. Lenné JM, Wood D. Is there a logic of fodder legumesin Africa? Food Policy. 2004;29:565-85.

38. Minson DJ. Forage in ruminant nutrition. Wisconsin - Madison: Academic Press; 1990. p. 483.

39. Soest VPJ. Nutritional Ecology of the ruminant. 2nd ed. Ithala, N.Y.: Cornel University Press; 1994.

40. Leal IR, Vicente A, Tabarelli M. Herbivoria por caprinos na Caatinga da região de Xingó: uma análise preliminar. In: Leal IR, Tabarelli M, Silva JMC, editors. Ecologia e conservação da caatinga. Recife: Ed. Universitária da UFPE; 2003.

41. Khanal ARC, Subba DB. Nutritional evaluation of leaves from some major fodder trees cultivated in the hills of Nepal. Animal Feed Science and Tecnology. 2001;92:17-32.

42. Moreira JN, Lira MA, Santos MVF. Caracterização da vegetação de Caatinga e da dieta de novilhos no Sertão de Pernambuco. Pesquisa Agropecuária Brasileira. 2006:41:1643-51.

43. Carvalho MVM, Ferreira RLC, Santos MVF, Dubeux Júnior JCB, Freitas AMM, Almeida OC. Caracterização de propriedades rurais e identificação de espécies arbóreas e arbustivas em pastagens do Agreste de Pernambuco. Revista Científica Produção Animal. 2001;3(1):38-54.

44. Bain JH. Mexican Rural Women's Knowledge of the environment. Mexican Studies. 1993:9:259-74

45. Hanazaki N, Tamashiro JY, Leitão-Filho HF, Begossi A. Diversity of plant uses in two Caiçara communities from the Atlantic Forest coast, Brazil. Biodivers Conserv. 2000;9:597-615.

46. Murrieta RSS, WinklerPrins MGA. Flowers of water: homegardens and gender roles in a riverine cabloco community in the lower Amazon, Brazil. Cult Agr. 2003;25(1):35-47.

47. Voeks RA. Are women reservoirs of traditional plant knowledge? Gender, ethnobotany and globalization in northeast Brazil. Singapore J Trop Geogr. 2007;28:7-20.

doi:10.1186/1746-4269-11-12

Cite this article as: Nunes et al.: Local knowledge about fodder plants in the semi-arid region of Northeastern Brazil. Journal of Ethnobiology and Ethnomedicine 2015 11:12

\section{Submit your next manuscript to BioMed Central and take full advantage of:}

- Convenient online submission

- Thorough peer review

- No space constraints or color figure charges

- Immediate publication on acceptance

- Inclusion in PubMed, CAS, Scopus and Google Scholar

- Research which is freely available for redistribution 